\title{
Management of a Delivery Suite During the COVID-19 Epidemic
}

\author{
Hongbo Qi ${ }^{1}$, Miao $\mathrm{Chen}^{2}$, Xin $\mathrm{Luo}^{1}$, Xiyao Liu ${ }^{1}$, Yuan $\mathrm{Shi}^{3}$, Tianjiao Liu ${ }^{1}$, Hua Zhang ${ }^{4}$, \\ Jun Zhang ${ }^{5}$, Yangyu Zhao ${ }^{2}$, Chao Tong ${ }^{2}$, and Philip Baker ${ }^{6}$ \\ ${ }^{1}$ Chongqing Medical University First Affiliated Hospital \\ ${ }^{2}$ Affiliation not available \\ ${ }^{3}$ Chongqing Medical University Affiliated Children's Hospital \\ ${ }^{4}$ The First Affiliated Hospital of Chongqing Medical University, Canada-China-New \\ Zealand Joint Laboratory of Maternal and Fetal Medicine, Chongqing Medical University \\ ${ }^{5}$ Xinhua Hospital, School of Medicine, Shanghai Jiao Tong University \\ ${ }^{6}$ University of Leicester
}

April 28, 2020

Since December 2019, the Severe Acute Respiratory Syndrome Coronavirus 2(SARS-CoV-2)has swept 200 countries and regions worldwide ${ }^{1}$ and has become a "Public Health Emergency of International Concern" (PHEIC). Pregnant women are susceptible to COVID-19 due to the changes in their physiology and the adaptability of their immune system ${ }^{2}$. During the outbreak of COVID-19, prenatal examinations may be postponed, however, delivery cannot be delayed, and the delivery room should work as usual. During this period, it is particularly important to quickly identify high-risk groups and to provide appropriate protection for childbirth and the puerperium. In accord with experience in China (Guidelines for the Prevention and Control of New Coronavirus Infections in Medical Institutions issued by the National Health Commission ), we strongly recommend that during the outbreak of COVID-19, all medical institutions should conduct graded, staged, comprehensive and continuous training of all staff, based on the particular epidemic prevention and control needs of for of different positions, to constantly improve staff's awareness of the prevention and control of COVID-19. To strengthen staff comprehension of the necessary precautions during a COVID19 epidemic, an assessment method that combines theory with scenario testing should also be applied ${ }^{3,4}$. At the same time, based on our experience of delivery room management, we recommend a delivery room processing flow (Fig.1) and graded protection ${ }^{5}$ (Table 1) for pregnant women with different infection risks,as detailed below:

(1) Primary screening of all women (First level protective equipment should be applied): Check the axillary temperature and the fetal heart rate, and enquire whether there is fever, respiratory symptoms (cough, chest tightness, etc.), gastrointestinal symptoms (vomiting, diarrhea, etc.) and other symptoms before allowing women to sit in the maternity waiting area. Ask whether there is increased risk of contact with a COVID-19 positive patient (fever of any family member within two weeks, a history of traveling to the epidemic area or contact with a suspected or confirmed patient). Any positive history of the above indicates 'potential risk' status.

(2) Pregnant women with potential-risk and/or suspected infection merit further screening (Second level protective equipment should be applied): attending staff should immediately apply second or third level of protective equipment, screening tests (which include respiratory pathogens tests like adenovirus, respiratory syncytial virus, influenza A virus, influenza B virus and parainfluenza virus, Mycoplasma pneumoniae and Chlamydia pneumoniae, blood routine tests, and C-reactive protein) should be undertaken and the new 
coronavirus nucleic acid test for pregnant women with potential-risk/suspected infection should be performed. A chest CT scan with informed consent to observe the lungs should be performed if signs or symptoms provide any indication (inform the patients about the necessity of chest CT and ask them to cover their abdomen properly). Obstetric management should not be delayed by testing for COVID-19.

(3) Delivery room management (for vaginal delivery): Pregnant women suspected to be COVID-19 positive should be immediately transferred to an isolated delivery room (avoiding contact with other patients) or negative pressure delivery room and be required to wear surgical mask ${ }^{6}$. Accompanying family must not be permitted. Patients should be managed by specific experienced senior medical specialists, and third level protective equipment must be applied to avoid cross-infection; pregnant women at potential risk of infection: Accompanying family should not be allowed. Patients are recommended to wear surgical masks ${ }^{6}$ and should be transferred to isolated delivery rooms, with management/supervision by specific experienced senior medical specialists. Second level protective equipment should be applied to prevent cross-infection, if availability of protective materials is adequate. low-risk pregnant women (those without any history of epidemiological exposure or clinical symptoms) should be transferred to an ordinary delivery room for delivery (avoiding contact with other patients). Second level protective equipment should be applied. It is recommended that these women wear disposable medical masks ${ }^{6}$. Only family members who have no history of epidemiological contact and clinical symptoms within the past 2 weeks are allowed to attend the childbirth, and accompanying family members are also required to wear disposable medical masks.

As fetal compromise is relatively common in pregnancies complicated by COVID-19 infection, continuous electronic fetal monitoring in labor is recommended for all women suspected with COVID-19, following transfer to the appropriate delivery room. ${ }^{7}$

We advocate attempts to deliver vaginally without undue obstetric intervention and recommend caution regarding procedures such as episiotomy and ventouse/forceps delivery. Currently, we do not recommend water deliveries for pregnant women with suspected infection. There is no evidence that epidural analgesia or spinal anesthesia is contraindicated, therefore, epidural analgesia should be recommended to pregnant women suspected of COVID-19 infection before or in early labor to minimize the need for general anesthesia in emergency situations ${ }^{7}$.

(4) Emergency caesarean section treatment:Suspected COVID-19 infection is not an indication for cesarean section, unless the woman's respiratory condition demands urgent delivery, or pregnant women have other indications. Multi-disciplinary consultation involving anaesthetists, neonatologists, obstetricians, and infectious disease physicians is required before deciding to deliver prematurely in cases of suspected infection, and if Caesarean section is indicated, the procedure should be performed in a negative pressure isolation operating room (third level protective equipment should be applied). The choice of anesthetic mode is determined by the anaesthetist, based on the patient's respiratory function. For pregnant women with potential infection (potential-risk), their pregnancy can be terminated in the isolated operating room (second level protective equipment should be applied) if properly protected. First level protective equipment is recommended when performing cesarean section for pregnant women with low-risk infection.

(5) Postpartum management: postpartum vital signs, uterine contractions, maternal mental health and other conditions of the mother should be monitored, and attention paid to the prevention of postpartum hemorrhage, thrombosis, etc. For pregnant women with suspected infection, the neonatologist should be notified at least half an hour before delivery to take appropriate measures to isolate the newborn. Delayed cord clamping is still recommended given a lack of evidence to the contrary, unless there are other contraindications ${ }^{7}$. 14 days of isolation for newborns is recommended ${ }^{8}$; there is currently no evidence to support the suspension of breastfeeding in pregnant women with suspected infection, indeed, we advocate breastfeeding, as the wider benefits outweigh the potential risks of transmission through breastmilk ${ }^{7}$. Isolation and preventive measures should be undertaken if referral is needed ${ }^{5}$. If there are no abnormal signs/symptoms within two hours after delivery, mothers with suspected infection can be transferred to an isolation ward for further observation; 'potential-risk' pregnant women can be transferred to the isolation ward (avoiding contact with other patients) and low-risk mothers managed according to conventional procedures. Pregnant woman with 
suspected or potential infection should undergo diagnostic testing immediately. If infection is confirmed, the corresponding management should follow the previous guidelines for dealing with confirmed cases of COVID-192.

(6) After-delivery protection procedures: After the mother was transferred to the ward, routine cleaning should be undertaken. The surfaces of the equipment (including the obstetric table, ultrasound machine, and neonatal warm bed) in the isolation delivery room and the negative-pressure delivery room need to be wiped and disinfected immediately, preferably with $1000 \mathrm{mg} / \mathrm{L}$ chlorine-containing disinfectant; $75 \%$ ethanol can be used for the non-corrosion resistance instruments ${ }^{7,9}$. Spraying is not a recommended method of disinfecting the equipment as this can affect the components. Dedicated cleaning tools are required to avoid cross contamination. The inspection room should be disinfected with ultraviolet light, [?]60 min each time, once or twice a day, with at least 30 min ventilation after irradiation. The ultrasound probe should be protected with a dark cloth during the irradiation. The room should be vacated when ultraviolet lamps are used.

(7) Medical waste disposal: Protective supplies used by medical personnel and all patient waste should be regarded as infectious medical waste, which requires double-layer sealing, clear labeling, and airtight transport ${ }^{10}$. If testing of the placenta and/or amniotic fluid is required, strict sampling and sealing should be carried out to avoid contamination of the surface of the container and the spread of infection. The surface of the container should be disinfected before sample inspection to further avoid infection of any personnel.

\section{Funding}

None.

\section{Ethics Approval}

Not required.

\section{Contribution to Authorship}

H.Q, C.T, and P. B conceived this study, H.Q, M.C, X.L, and C.T wrote the draft, X.L, T.L, H.Z, J.Z, Y.Z, Y.S and P. B revised the manuscript.

\section{Disclosure of Interests}

None declared.

\section{Reference}

1. Alexander EG, Susan CB, Ralph SB, Raoul JG, Christian D, Anastasia AG, et al. Severe acute respiratory syndrome-related coronavirus: The species and its viruses - a statement of the Coronavirus Study Group.BioRxiv 2020. https://doi.org/10.1101/2020.02.07.937862

2. Qi H, Luo X, Zheng Y, Zhang H, Li J, Zou L, et al. Safe Delivery for COVID-19 Infected Pregnancies. BJOG 2020. https:// doi.org /10.1111/1471-0528.16231

3. National Health Commission of the People's Republic of China. Technical guidelines for the prevention and control of COVID-19 infection in medical institutions -first edition [Internet]. 2020. Available from: http://www.nhc.gov.cn/yzygj/s7659/202001/b91fdab7c304431eb082d67847d27e14.shtml

4. National Health Commission of the People's Republic of China. Notice of the General Office of the National Health Commission on further strengthening the prevention and control of infections in medical institutions during the Epidemic [Internet]. 2020. Available from: http://www.nhc.gov.cn/xcs/zhengcwj/202003/0c859

5. Li C, Huang X, Cai M, Chen P, Chen W, Chen X, et al. Expert consensus on personal protection in different regional posts of medical institutions during COVID-19 epidemic period. Chin J Infec Contl. 2020;19(3):199-214.

6. National Health Commission of the People's Republic of China. Notice on issuing technical guidelines for the selection and instruction of masks for prevention of COVID-19 infection in different populations [Internet]. 2020. Available from: http://www.nhc.gov.cn/jkj/s7916/202002/485e5bd019924087a5614c4f1db135a2.shtm 
7. RCOG. Coronavirus (COVID-19) infection and pregnancy. Royal College of Obstetricians and Gynaecologists. 2020. [https://www.rcog.org.uk/coronavirus-pregnancy]. Accessed 28 March 2020.

8. Wang J, Qi H, Bao L, Li F, Shi Y. A contingency plan for the management of the 2019 novel coronavirus outbreak in neonatal intensive care units. Lancet Child Adolesc Health. 2020; pii: S23524642(20)30040-7.

9. Health Protection Scotland. COVID-19 - guidance for secondary care. National Services Scotland. 2020. [https://www.hps.scot.nhs.uk/web-resources-container/covid-19-guidance-for-secondary-care/]. Accessed 06 March 2020.

10. National Health Commission of the People's Republic of China. Notice on completion of medical waste management in medical institutions during the pneumonia epidemic of COVID-19 infection [Internet]. 2020. Available from: http://www.gov.cn/zhengce/zhengceku/2020-01/28/content_5472796.htm

Table 1: List of protection levels andprotective equipment

\begin{tabular}{llll}
\hline Protective equipment & Protection levels & Protection levels & Protection levels \\
\hline & First level & Second Level & Third Level \\
Disposable work cap & $*$ & $*$ & $*$ \\
Medical surgical mask & $*$ & & $*$ \\
Medical protective mask & $*$ & $*$ & $*$ \\
Protective goggle/face shield & & $*$ & $*$ \\
Positive pressure breathing hood & $*$ & $*$ & $*$ \\
Scrubs & $*$ & & $*$ \\
Disposable isolation suit & $*$ & $*$ & $*$ \\
Disposable protective suit & & $*$ & $*$ \\
Disposable latex glove & $*$ & $*$ & $*$ \\
Disposable shoes cover & & $*$ & $*$ \\
Hand hygiene & $*$ & & \\
\hline
\end{tabular}

Note: *mandatory *when necessary

(1) In first-level protection: medical surgical masks and medical protective masks should not be used at the same time;

(2) In third-level protection: medical protective masks and protective goggle/face shield are unnecessaty if positive pressure headgear is used.

Figure 1: The Processing Flow of Delivery During the COVID-19 Outbreak Based on the Risk Level of Pregnancy Infection. 


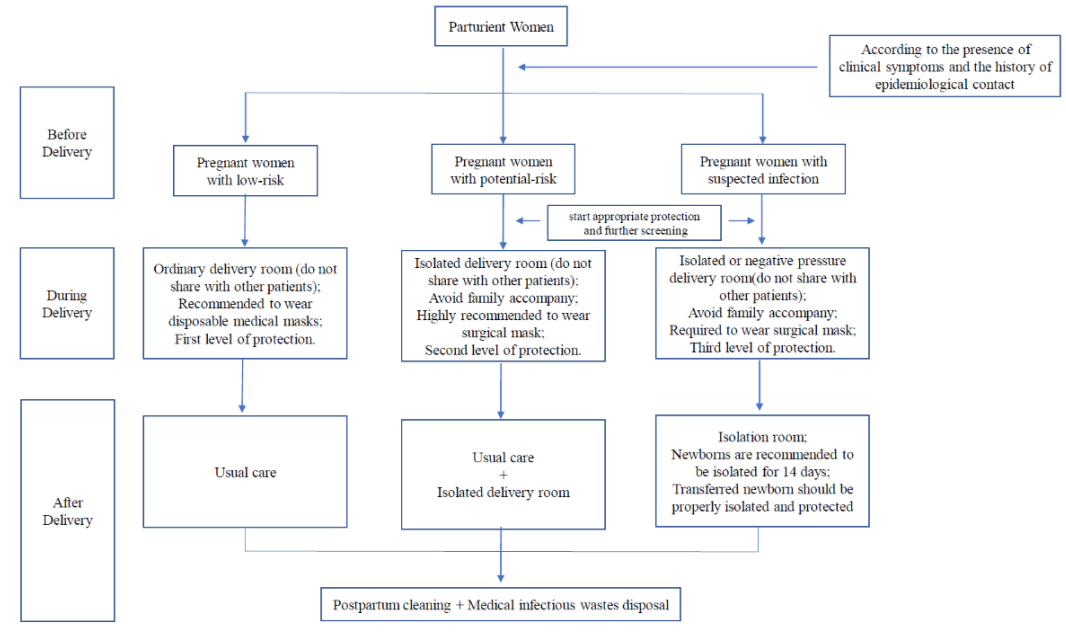

